Beatriz Maykot Kuerten Gil. ${ }^{1}$

EDUARDO DE SOUZA ${ }^{2}$

Carlos Alberto Justo da Silva ${ }^{3}$

Cláudia Pinto Figueiredo ${ }^{4}$

Artigo original

Palavras-chave

Maturidade dos órgãos fetais Líquido amniótico

Surfactantes pulmonares

Técnicas de diagnóstico e procedimentos

Síndrome do desconforto respiratório do recém-nascido

Keywords

Fetal organ maturity Amniotic fluid

Pulmonary surfactants Diagnostic techniques and procedures Respiratory distress syndrome, newborn

\section{Avaliação da maturidade pulmonar fetal pela contagem dos corpos lamelares no líquido amniótico}

\author{
Evaluation of fetal lung maturity by lamellar bodies counting in \\ amniotic fluid
}

\section{Resumo}

OBJETIVO: comparar o teste de contagem de corpos lamelares (CCL) no líquido amniótico com o teste da polarização fluorescente (PF) como parâmetro diagnóstico para avaliação da maturidade pulmonar fetal. MÉTODO: estudo transversal, analítico e controlado realizado com 60 gestantes atendidas no período de março de 2002 a dezembro de 2007. Foram colhidas amostras de líquido amniótico e realizados os testes de CCL e PF (TDxFLM II), considerados de referência, e comparados à presença ou ausência da Síndrome do Desconforto Respiratório (SDR). Foram estabelecidos valores de corte para maturidade de 30 mil corpos lamelares/ $\mu \mathrm{L}$ para o teste da CCL e $55 \mathrm{mg} / \mathrm{g}$ de albumina para o PF. Foram avaliadas as características maternas e perinatais, a evolução neonatal e o desempenho dos testes diagnósticos para predição da maturidade pulmonar fetal. Na análise estatística, foram utilizadas medidas descritivas e calculados os valores referentes à sensibilidade, especificidade, valor preditivo positivo e negativo dos testes, considerando-se significativos valores de p $\leq 0,05$. RESULTADOS: a idade materna variou entre 15 e 43 anos, com média de 26,6 anos. A idade gestacional variou entre 24,3 e 41,6 semanas, com média de 35, 1 semanas. A Síndrome do Desconforto Respiratório foi diagnosticada em 13,3\% dos neonatos. As características perinatais, como peso, índice de Apgar, incidência de SDR, foram comparadas aos resultados dos testes de CCL e PF, sendo observada uma correspondência, estatisticamente significativa $(p \leq 0,05)$, entre os grupos de neonatos clinicamente classificados como imaturos e maduros em ambos os testes. Os testes foram concordantes em $68,3 \%$ dos casos. Quando se comparou o teste da PF com o teste da CCL, a sensibilidade foi de 100\% para ambos, e a especificidade do teste da CCL foi superior $(73,1 \%)$, quando comparado com o teste de PF (51,9\%). O padrão-ouro para determinação da maturidade fetal é a ocorrência da SDR. $\bigcirc$ valor preditivo positivo do teste da CCL foi superior $(36,4 \%)$ quando comparado ao teste da PF $(24,2 \%)(p<0,05)$, sendo que o valor preditivo negativo foi de $100 \%$ para ambos os testes. CONCLUSÕES: este estudo demonstrou que o teste da CCL apresenta 100\% de sensibilidade e especificidade superior ao teste de referência (PF). Além disso, a CCL é considerada um teste rápido, acessível, barato e factível em nossa realidade, podendo ser utilizado como teste confiável na predição da maturidade pulmonar fetal.

\section{Abstract}

PURPOSE: to compare the lamellar body number density (LBND) count in amniotic fluid using the fluorescent polarization (FP) test as a diagnostic parameter for the assessment of fetal pulmonary maturity. METHOD: this was an analytical, controlled cross-sectional study conducted on 60 pregnant women from March 2002 to December 2007. Amniotic fluid specimens were obtained by amniocentesis or at the time of caesarean section, and submitted to the LBND and FP tests (TDxFLM ${ }^{\circledast}$, Abbott Laboratories), the latter considered to be a reference test, and compared in terms of the presence or absence of respiratory distress syndrome (RDS). Cut-off values for maturity were established at 30,000 lamellar bodies/ $\mu \mathrm{L}$ for the LBND test and $55 \mathrm{mg} / \mathrm{g}$ albumin for the FP test. Maternal and perinatal characteristics and neonatal evolution were evaluated, and the performance of the diagnostic tests regarding fetal pulmonary maturity was determined. In the statistical analysis, descriptive measures were used and the sensitivity, specificity and positive and predictive values of the tests were determined with the level of significance set at $p \leq 0.05$. RESULTS: maternal
Correspondência:

Beatriz Maykot Kuerten Gil Universidade Federal de Santa Catarina Hospital Universitário, Departamento de Tocoginecologia Campus Universitário - Bairro Trindade CEP 88040-970 - Florianópolis (SC), Brasil E-mail:beakuerten_@hotmail.com
Departamento de Tocoginecologia da Universidade Federal de Santa Catarina - UFSC - Florianópolis (SC), Brasil.

Professora do Departamento de Tocoginecologia e médica do Serviço de Ginecologia e Obstetrícia do Hospital Universitário da Universidade Federal de Santa Catarina - UFSC - Florianópolis (SC), Brasil.

2 Professor Titular do Departamento de Obstetrícia da Universidade Federal de São Paulo - UNIFESP - São Paulo (SP), Brasil. ${ }^{3}$ Professor do Departamento de Clínica Cirúrgica da Universidade Federal de Santa Catarina - UFSC - Florianópolis (SC), Brasil. ${ }^{4}$ Pós-doutoranda do Programa de Pós-Graduação em Neurociências do Departamento de Ciências Fisiológicas, Centro de Ciências Biológicas da Universidade Federal de Santa Catarina - UFSC - Florianópolis (SC), Brasil. 
age ranged from 15 to 34 years (mean: 26.6 years) and gestational age ranged from 24.3 to 41.6 weeks (mean: 35.1 weeks). RDS was diagnosed in $35.1 \%$ of neonates. Perinatal characteristics such as weight, Apgar score, and RDS incidence were compared to the results of the LBND and FP tests and a significant correspondence ( $\mathrm{p} \leq 0.05)$ was observed between the groups of neonates clinically classified as mature and immature in both tests. The tests were concordant in $68.3 \%$ of the cases. Comparison of the PF and LBND tests revealed $100 \%$ specificity for both and a higher specificity for the LBND test $173.1 \%$ as opposed to $51.9 \%$ for the PF test). The gold standard for the determination of fetal maturity is the occurrence of RDS. The positive predictive value of the LBND test was higher (36.4\%) than that of the FP test $(24.2 \%)(p<0.05)$ and the negative predictive value was $100 \%$ for both tests. CONCLUSIONS: the present study demonstrated that the LBND test has 100\% sensitivity and higher specificity than the reference test (FP). In addition, the LBND test is considered to be rapid, accessible, inexpensive and feasible for the Brazilian reality, and it can be used as a reliable test for the prediction of fetal pulmonary maturity.

\section{Introdução}

Em várias situações obstétricas, sobretudo naquelas conhecidas como gestações de alto risco, nas quais existem condições clínicas adversas em que o nascimento do feto antes do termo pode representar a necessidade de intervenção terapêutica imperativa e valiosa ${ }^{1,2}$. $\mathrm{O}$ risco do parto prematuro constitui um dos maiores desafios na prática obstétrica, e o conhecimento da maturidade pulmonar fetal torna-se de vital importância, visto que a Síndrome do Desconforto Respiratório (SDR) é uma das principais causas de morbimortalidade neonatal ${ }^{1-5}$.

A presença dos fosfolipídios, lecitina, esfingomielina, fosfatidilinositol, fosfatidilglicerol e outras substâncias no líquido amniótico (LA), além da demonstração de que os mesmos eram provenientes do pulmão fetal, direcionou as pesquisas para a predição da maturidade pulmonar fetal ${ }^{1,3,6}$.

O pulmão fetal inicia seu desenvolvimento por volta da terceira semana de vida e, próximo da $24^{a}$ semana de gestação, algumas células que revestem os ácinos diferenciam-se em pneumócitos do tipo I e II. O pneumócito do tipo I é uma célula com funções principalmente relacionadas ao revestimento alveolar. Já o pneumócito do tipo II é célula rica em corpos lamelares (CL), que são estruturas intracelulares com 1 a $5 \mu$ de diâmetro, ricas em lipídios e que armazenam as substâncias surfactantes. As substâncias surfactantes são observadas entre a $24^{\mathrm{a}}$ e a $26^{a}$ semana gestacional, sendo que ao final deste período o pulmão fetal já apresenta alguma capacidade de realizar trocas gasosas, embora ainda imaturo ${ }^{7}$. Alterações na proporção, na quantidade ou na qualidade dos fosfolipídios que compõem o surfactante pulmonar resultam em colapso alveolar, ocasionando a SDR, também conhecida como doença pulmonar das membranas hialinas com consequente atelectasia progressiva, edema, alteração da relação ventilação/perfusão, levando à hipóxia tecidual ${ }^{3,4,6}$.

O primeiro teste descrito para avaliar o grau de maturidade pulmonar fetal, a relação lecitina/esfingomielina (L/E), é utilizado até os dias atuais, porém não é realizado em vários centros devido ao alto custo e à tecnologia envolvida ${ }^{7}$. O teste de Clements, um dos primeiros métodos utilizados para predição da maturidade pulmonar, é considerado um teste rápido, com baixo custo e de fácil execução. Entretanto, a ocorrência de resultados falsonegativos, apesar da controvérsia, parece relativamente alta $^{2,7,8}$. Cunningham et al. acreditam que é necessária uma relação L/E de 4,0 ou mais para produzir um teste de Clements positivo 9 , enfatizando que as amostras contaminadas com sangue e/ou mecônio, ou mesmo a presença de contaminantes externos (detergentes e líquidos biológicos) nas vidrarias, podem interferir nos resultados do teste ${ }^{1}$, gerando resultados falso-positivos ${ }^{10}$. Além disso, um estudo realizado em 2006 com 62 pacientes concluiu que a CCL tem melhor desempenho quanto à especificidade, valor preditivo positivo e acurácia quando comparado ao teste de Clements ${ }^{11}$.

O método da contagem dos corpos lamelares foi introduzido em $1989^{12}$ como uma alternativa viável para predição da maturidade pulmonar fetal. Os corpos lamelares são reservatórios de fosfolipídios, componentes do surfactante pulmonar, que são levados ao LA por meio do movimento respiratório fetal. A técnica consiste na contagem dos corpos lamelares em amostras de LA utilizando contadores hematológicos ${ }^{12-14}$, que têm se tornado cada vez mais comuns nos laboratórios de análises clínicas. A determinação do número de corpos lamelares presentes no LA é realizada utilizando-se o mesmo parâmetro de ajuste para contagem de plaquetas (2-20 fL), pois os corpos lamelares possuem volume médio entre 1,7-7,3 fL, semelhante ao das plaquetas, o que os torna facilmente reconhecíveis pelo aparelho ${ }^{3}$.

O teste da CCL já foi sugerido por alguns autores como teste de rastreamento de maturidade pulmonar por apresentar sensibilidade, especificidade, VPP e VPN comparáveis aos testes de referência ${ }^{3,7}$. Além disso, podemos destacar outros benefícios, como o baixo custo, rapidez, fácil execução e necessidade de pequeno volume de amostra; sendo assim, tem sido sugerido como teste de rastreamento na predição da maturidade pulmonar fetal $^{1,7,13-15}$. Entretanto, a utilização da CCL no Brasil ainda é pouco difundida e há poucas citações na literatura.

O objetivo deste estudo foi comparar o teste de CCL com o teste da PF, considerado um teste de referência, como uma alternativa mais rápida, acessível, barata e específica para determinação da maturidade pulmonar fetal. 


\section{Métodos}

Foi realizado um estudo transversal analítico e controlado com gestantes do Serviço de Obstetrícia da Maternidade do Hospital Universitário (UFSC) e da Clínica e Maternidade Saint Patrick, Florianópolis (SC), no período de março de 2002 a outubro de 2007. O estudo foi aprovado pelo Comitê de Ética em Pesquisas com Seres Humanos da Universidade Federal de Santa Catarina, (processo 001/2002), estando de acordo com as Diretrizes e Normas Regulamentadoras de Pesquisa envolvendo Seres Humanos, Resoluções 196/96 e 251/97 do Conselho Nacional de Saúde.

A amostra foi constituída por 74 gestantes internadas no Hospital Universitário $(\mathrm{n}=52)$ e na Clínica e Maternidade Saint Patrick $(n=22)$. Dessas, 14 foram excluídas por não obedecerem aos critérios de inclusão desta pesquisa, restando 60 gestantes na amostra. A amostragem foi do tipo intencional, não-probabilística, constituída por todos os casos internados na Enfermaria de Alto Risco no período compreendido pela pesquisa. Estes casos foram encaminhados para avaliação da maturidade pulmonar fetal. As amostras de LA foram obtidas por meio de amniocentese transabdominal ou no momento da cesariana quando consentido pela paciente.

Os critérios de inclusão foram presença de feto vivo, único, idade gestacional entre 24 e 42 semanas, intervalo inferior a 72 horas entre amniocentese, análise do LA e parto. A idade gestacional foi calculada a partir do primeiro dia da última menstruação e confirmada por ultrassonografia obstétrica ou Capurro somático. O método de New Ballard foi utilizado nos casos de idade gestacional inferior a 34 semanas, quando o Capurro é contraindicado.

Os critérios de exclusão foram corioamnionite, contaminação do LA por sangue e/ou mecônio, recém-nascido com desconforto respiratório associado a outras causas que não a imaturidade pulmonar, malformações fetais congênitas maiores, doenças pulmonares ou cardíacas do recém-nascido determinantes de desconforto respiratório e que pudessem interferir na análise dos resultados.

Nos casos de amniocentese transabdominal, o procedimento foi realizado por meio de equipamento da marca $\mathrm{GE}^{\circledR}$ modelo RT 4600, com transdutor de tempo real convexo de 3,5 MHz. Realizou-se a punção com agulha descartável, com ponta, tipo Quincke $22 \mathrm{G}$ (Gauge) da marca BD ${ }^{\circledR}$, sob visão ultrassonográfica direta. Para aspiração do LA, foi utilizada seringa plástica descartável de $20 \mathrm{~mL}$ (Luer Slip) da marca BD Plastipak ${ }^{\circledR}$, Nos casos em que o LA foi obtido no momento da cesárea, após a histerotomia segmentar, ainda com as membranas íntegras foi puncionada a cavidade amniótica com agulha estéril descartável $21 \mathrm{G} 1 / 4$, medindo $0,80 \mathrm{x}$ $30 \mathrm{~mm}$, marca $\mathrm{BD}^{\circledR}$, e o LA foi aspirado com seringa de $20 \mathrm{~mL}$, com as mesmas características daquela utilizada na amniocentese. Em ambos os procedimentos foram coletadas duas amostras de LA em punção única, com seringas separadas, contendo cada uma no mínimo $3 \mathrm{~mL}$ de LA. Ambas foram fechadas com agulhas descartáveis, encapadas e esterilizadas, envoltas em papel carbono para evitar a exposição à luz, visto que o teste FP de referência (TDxFLM ${ }^{\circledR}$ ) é fotossensível, e identificadas para posterior análise.

No laboratório de análises clínicas do Hospital Universitário, foi realizado o teste CCL e o teste FP foi encaminhado para um laboratório externo, que mantinha convênio com a instituição. Este último tinha a finalidade de obter um controle ao teste CCL. Para a CCL, utilizouse contador hematológico da marca Coulter T-890 ${ }^{\circledR}$, requerendo-se $0,5 \mathrm{~mL}$ de LA por amostra processada. As amostras eram misturadas através de delicada inversão e colocadas em tubos plásticos descartáveis de $12 \times 75 \mathrm{~mm}$, centrifugadas (Centrífuga Fanem modelo 206- $\mathrm{R}^{\circledR}$ ) durante três minutos a $2.000 \mathrm{rpm}(500 \mathrm{~g})$, com a finalidade de remover restos celulares. O sobrenadante foi colocado em outro tubo plástico, com as mesmas medidas do anterior, e levado ao contador, onde era automaticamente aspirado $0,18 \mathrm{~mL}(180 \mu \mathrm{L})$ para realização da contagem, através do princípio da emissão de pulsos elétricos ${ }^{12}$. Os parâmetros que o equipamento utilizou para a CCL são similares aos da contagem de plaquetas em amostras de sangue total. Conforme descrito anteriormente, o canal de contagem de plaquetas do equipamento avalia partículas com volume entre 2-20 fL, sendo que os $\mathrm{CL}$ possuem volume entre 1,7-7,3 fL. O tempo médio para execução do teste foi de aproximadamente 15 minutos. Foi caracterizada como prova negativa, ou seja, amostra positiva para maturidade pulmonar fetal, aquela cuja contagem dos corpos lamelares foi inferior a 30.000 partículas $/ \mu \mathrm{L}$, e prova positiva aquela cujo resultado foi igual ou superior a 30.000 partículas $/ \mu \mathrm{L}$.

A avaliação da relação surfactante/albumina foi realizada com o equipamento de análise do teste de PF (TDxFLx Abbott $^{\circledR}$, Abbott Laboratories, Abbott Park, II), conforme recomendações fornecidas pelo fabricante. Foi considerada prova negativa, ou seja, amostra positiva para maturidade pulmonar fetal aquela cujo resultado for inferior a $55 \mathrm{mg} / \mathrm{g}$ de albumina, e prova positiva aquela cujo resultado foi igual ou superior a $55 \mathrm{mg} / \mathrm{g}$ albumina.

O desempenho dos testes diagnósticos foi comparado com a presença ou ausência da SDR no recém-nascido, considerado o padrão-ouro para estabelecimento de imaturidade pulmonar fetal ${ }^{7}$. A SDR foi diagnosticada baseando-se nos critérios clínicos, na gasometria arterial, achados radiológicos e suplementação de oxigênio (superior a 48 horas) ${ }^{4}$. A padronização da avaliação neonatal se manteve porque os neonatologistas da Clínica Saint Patrick são comuns ao Serviço de Neonatologia do Hospital Universitário. 
Para a análise estatística, as medidas descritivas empregadas foram média, desvio padrão (DP) e tabelas de contingência. Para comparar as frequências das características perinatais com a classificação de imaturo e maduro segundo o teste CCL e PF, utilizou-se o teste de proporções. A variável de análise (maturidade) foi dicotomizada e foram construídas tabelas de contingência para determinar a associação dos resultados dos testes CCL e $\mathrm{PF}$, com a presença ou não da SDR, utilizando-se o teste $\chi^{2}$ de Pearson para um nível de significância de $5 \%$. As tabelas $2 \times 2$ foram montadas para cálculos de sensibilidade (S), especificidade (E), valor preditivo positivo (VPP) e valor preditivo negativo (VPN). Para verificar se existe diferença significativa entre as especificidades dos dois testes diagnósticos, utilizou-se o teste de McNemar. Foram considerados significativos valores de $\mathrm{p} \leq 0,05$.

\section{Resultados}

A idade das gestantes inclusas no estudo foi de 15 a 43 anos (média de 26,6 $\pm 6,9$ anos). A idade gestacional variou entre 24,3 e 41,6 semanas (média de 34,8 44,56 semanas). Quando utilizada a idade gestacional para predição da imaturidade pulmonar e comparada ao resultado dos testes de CCL e PF, observou-se menor concentração de resultados falso-positivos quando utilizado o teste de CCL.

A Tabela 1 demonstra as características perinatais e a classificação entre maduros e imaturos de acordo com a contagem de corpos lamelares. Foi possível observar que o peso médio encontrado no grupo cujo CCL era inferior $30.000 / \mu \mathrm{L}$ (imaturos) foi de $1.519 \mathrm{~g}$, em contrapartida ao grupo de recém-nascidos com contagem superior a $30.000 / \mu \mathrm{L}$ (maduros). A quantidade de $\mathrm{RN}$ com muito baixo peso $(<1.500 \mathrm{~g})$ foi significativamente menor no grupo $<30.000 / \mu \mathrm{L}(\mathrm{p}<0,0001)$. Escores de Apgar $<7$, tanto no primeiro como no quinto minuto, foram significativamente menores no grupo $<30.000 / \mu \mathrm{L}$ quando comparados com o grupo $\geq 30.000 / \mu \mathrm{L}(\mathrm{p}=0,0005)$. A presença de SDR foi significativamente superior no grupo cujo teste apontava resultado compatível com imaturidade $(\mathrm{p}=0,0002)$ (Tabela 1).

A Tabela 2 demonstra as características perinatais e a classificação entre maduros e imaturos de acordo com o resultado do teste PF. Observou-se que o peso médio encontrado no grupo classificado como imaturo foi de $1.938 \mathrm{~g}$, sendo que o grupo classificado como maduro foi de $2.989 \mathrm{~g}$. A quantidade de RN com muito baixo peso $(<1.500 \mathrm{~g})$ foi significativamente menor no grupo de imaturos $(\mathrm{p}=0,0104)$. Os grupos classificados como imaturos e maduros por meio do teste PF não apresentaram diferença significativa nas proporções do escore de Apgar $<7$ no primeiro minuto ( $p>0,05)$. Entretanto, o grupo de recém-nascidos classificados como imaturos apresentou uma maior proporção de escore de Apgar < 7 no quinto minuto quando comparado com o grupo classificado como maduro $(\mathrm{p}=0,0005)$. A presença de SDR foi significativamente superior no grupo cujo teste apontava resultado compatível com imaturidade $(\mathrm{p}=0,0002)$ (Tabelas 2 e 3$)$.

$\mathrm{Na}$ Tabela 3, dicotomizou-se a variável de análise, ou seja, a maturidade, sendo construída uma tabela de contingência para demonstrar a associação dos resultados dos testes de CCL e PF, mediante presença ou ausência da SDR. Estes resultados demonstram que, nesta amostra, o teste de CCL e PF apresentaram 100\% de especificidade (Tabelas 3 e 4). Pode-se notar que, em 41/60 (68,3\%)

Tabela 1 - Características perinatais de acordo com o teste contagem dos corpos lamelares (CCL)

\begin{tabular}{lcccc}
\hline Características perinatais & Imaturo & Maduro & Valor de p \\
\hline Peso cao nascer (média em gramas) & 1519 & 2927 & \\
Proporç̃o abaixo de $1.500 \mathrm{~g}$ & $11 / 22$ & $0 / 38$ & $0,0001^{\star}$ \\
Proporç̃óo de Apgar de 1 min $<7$ & $12 / 22$ & $3 / 38$ & $0,0002^{\star}$ \\
Proporç̃o de Apgar de 5 min $<7$ & $7 / 22$ & $0 / 38$ & $0,0005^{\star}$ \\
Incidência de SDR & $8 / 22$ & $0 / 38$ & $0,0002^{\star}$ \\
\hline
\end{tabular}

* diferença significativa, pelo teste de proporções, considerando o nível de significância de $5 \%$.

Tabela 2 - Características perinatais de acordo com o resultado do teste Polarização Fluorescente (PF)

\begin{tabular}{l|ccc}
\hline Características perinafais & Imafuro & Maduro & Valor de p \\
\hline Peso ao nascer (média em gramas) & 1938 & 2989 & \\
Proporção abaixo de $1.500 \mathrm{~g}$ & $10 / 33$ & $1 / 27$ & $0,0104^{\star}$ \\
Proporção de Apgar de 1 min $<7$ & $8 / 26$ & $3 / 34$ & \\
Proporção de Apgar de 5 min $<7$ & $13 / 33$ & $2 / 27$ & $0,0005^{\star}$ \\
Incidência de SDR & $8 / 33$ & $1 / 27$ & $0,0002^{\star}$ \\
\hline
\end{tabular}

*Diferença estatística, pelo teste de proporções, considerando o nível de significância de $5 \%$.

Tabela 3 - Diagnóstico da Síndrome do Desconforto Respiratório relacionado com a classificacão da maturidade pulmonar fetal por meio dos testes de CCL e PF

\begin{tabular}{llccc}
\hline \multirow{2}{*}{ Testes } & \multicolumn{3}{c}{ SDR } & Total \\
\cline { 3 - 5 } & & Presente & Ausente & \\
\cline { 3 - 5 } & & $\mathbf{n}(\%)$ & $\mathbf{n}(\%)$ & $\mathbf{n}(\%)$ \\
\hline \multirow{2}{*}{ CCL } & Maduro & $0(0,0)$ & $38(63,3)$ & $38(63,3)$ \\
& Imaturo & $8(13,3)$ & $14(23,3)$ & $22(36,7)$ \\
\multirow{2}{*}{ TDxFLM } & Maduro & $0(0,0)$ & $27(45,0)$ & $27(45,0)$ \\
& Imaturo & $8(13,3)$ & $25(41,7)$ & $33(55,0)$ \\
\hline
\end{tabular}

Tabela 4 - Valores de sensibilidade, especificidade, valor preditivo positivo (VPP), valor preditivo negativo (VPN) e a probabilidade do teste de McNemar entre os testes

\begin{tabular}{lcccc}
\hline Testes de diagnóstico & Sensibilidade & Especificidade & VPP & VPN \\
\hline CCL & $100 \%$ & $73,10 \%$ & $36,40 \%$ & $100 \%$ \\
TDxFLM & $100 \%$ & $51,90 \%$ & $24,20 \%$ & $100 \%$ \\
CCL versus TDxFLM & NS & $0,0367^{\star}$ & & \\
\hline
\end{tabular}

*Valor de $p$ significativo $(p \leq 0,05)$ usando o teste McNemar. NS representa o valor de $p$ não-significativo $(p>0,05)$ usando $\circ$ teste de $\mathrm{McNemar}$. 
recém-nascidos, os resultados entre os testes de CCL e PF foram concordantes entre si, sendo ambos positivos para maturidade em 23/60 (38,3\%) e para imaturidade em $18 / 60(30,0 \%)$. Os testes foram discordantes em 19/60 casos $(31,7 \%)$, sendo que em $15 / 19(78,9 \%)$ o PF foi negativo para maturidade enquanto o CCL foi positivo, o que demonstra uma maior concordância dos testes na ausência de imaturidade (Tabela 3).

Para a análise dos valores de predição dos métodos de CCL e PF, foram testados, além dos parâmetros de sensibilidade (S) e especificidade (E), o valor preditivo positivo (VPP) e o valor preditivo negativo (VPN). Quando utilizado o teste de McNemar, foi observada uma especificidade estatisticamente superior no teste $\operatorname{CCL}(73,1 \%)$ em relação ao teste $\operatorname{PF}(51,9 \%)(\mathrm{p}=0,0367)$ (Tabela 4). O VPN de ambos os testes foi de $100 \%$, sendo que o VPP foi de $36,4 \%$ para a CCL e $24,2 \%$ para PF, respectivamente (Tabela 4). Ou seja, a probabilidade de o teste ser negativo para imaturidade, sabendo-se que o recém-nascido examinado não é portador de SDR, é maior para o teste CCL do que para a PF. Sendo assim, ambos os testes mostraram-se sensíveis e com alto VPN, mas o CCL parece apresentar uma maior especificidade para o rastreamento da SDR.

\section{Discussão}

A prevenção da Síndrome do desconforto respiratório e todas as suas complicações por meio da obtenção de informações precisas e confiáveis a respeito do grau de maturidade pulmonar fetal tem sido a principal finalidade das pesquisas atuais nessa área $a^{5,6,14,16,17}$. O estabelecimento do momento mais oportuno para a realização do parto prematuro terapêutico é realizado diariamente, sendo essencial a disponibilidade de informações precisas sobre a incidência das principais intercorrências neonatais e a idade gestacional em que ocorrem para que o obstetra possa adotar as condutas adequadas.

A magnitude do tema está intimamente relacionada à morbidade e à mortalidade neonatal, fundamentalmente dos recém-nascidos prematuros, que representam cerca de 10 a $15 \%$ de todos os neonatos ${ }^{1}$. Entre as múltiplas complicações da prematuridade, a imaturidade pulmonar, relacionada à produção inadequada de surfactante, representa uma das mais graves, e que compromete com frequência a sobrevida do concepto ${ }^{7}$.

O diagnóstico clínico da maturidade pulmonar está relacionado com a idade gestacional, sendo que na gestação normal ocorre paralelismo entre o tempo de evolução do feto e a maturidade dos seus vários sistemas orgânicos, entre eles o pulmonar ${ }^{7}$. A SDR foi diagnosticada em 13,3\% (8 casos), sendo que a sua incidência na literatura varia entre 7 e $14 \%^{1,7}$. A maturidade do sistema respiratório fetal ocorre por volta da $35^{a}$ semana gestacional, quando as adaptações anatômicas e funcionais permitem ao recémnascido prematuro sobreviver no ambiente extrauterino ${ }^{3,4}$. A média da idade gestacional dos recém-nascidos com SDR foi de 30,3 semanas (dados não mostrados). Entretanto, em apenas um caso deste estudo, a SDR ocorreu em um neonato com idade gestacional de 35,5 semanas, sendo que todos os demais casos com idade gestacional superior a 35 semanas foram considerados casos de falso-positivos para imaturidade.

Comparamos os resultados obtidos nos testes de CCL e PF e observamos que ambos os testes foram capazes de selecionar os casos de imaturidade. Estes resultados confirmam com estudos anteriores que relatam uma maior ocorrência de imaturidade pulmonar em recém-nascidos com baixo peso e escores de Apgar $<7$ no primeiro e quinto minuto ${ }^{6}$. Nos casos de SDR encontrados em nosso estudo esses escores variaram entre 3 e 7 (média $=5$ ), enquanto no quinto minuto variaram entre 5 e 7 (média $=6$ ). Adicionalmente, a média do escore de Apgar dos recémnascidos maduros (sem SDR) foi de 7,8 e 8,8 no primeiro e no quinto minuto, respectivamente, confirmando os resultados deste estudo.

Entre as características perinatais, a ocorrência de SDR é considerada padrão-ouro para a validação dos testes de rastreamento de ocorrência de imaturidade pulmonar fetal ${ }^{7}$. É importante ressaltar que os oito casos de SDR diagnosticados neste estudo foram previamente classificados como imaturos pelos testes de CCL e PF, sendo que os valores da CCL variaram entre 6.000 e 9.000 partículas/ $\mu \mathrm{L}$ e a idade gestacional entre 24 e 35,5 semanas. Estes dados estão em concordância com outros estudos ${ }^{18,19}$ que definem que valores $\leq 10.000$ partículas $/ \mu \mathrm{L}$ são de alto risco para a ocorrência da SDR ${ }^{1,12}$.

Quando comparados os resultados obtidos nos dois testes de rastreamento, foram observadas discordâncias entre os grupos cujos neonatos foram classificados como imaturos e maduros de acordo com o teste CCL e PF. Estes dados demonstram que o teste da CCL apresentou uma capacidade melhor na discriminação dos fetos que realmente estavam imaturos, quando comparados ao teste referência (PF). Um número maior de casos foi classificado de forma incorreta como imaturos por meio do teste PF, o que caracteriza uma especificidade menor deste teste quando comparado à CCL.

A sensibilidade, ou seja, a capacidade de diagnosticar doença (SDR) foi de $100 \%$, e a especificidade, ou seja, a capacidade de excluir a doença, foi de $73,1 \%$. O VPP, também conhecido como sensibilidade diagnóstica, foi de $36,4 \%$, sendo concordante com um estudo de Dalence et al. ${ }^{1}$, que demonstrou em uma população de 130 pacientes a ocorrência de VPP de $28 \%$ para o CCL. O VPN, também conhecido como especificidade diagnóstica, foi 
de $100 \%$, e isso significa que quando o teste apresentou resultado positivo para maturidade, a doença realmente não ocorreu, ou seja, trata-se de um resultado altamente expressivo na identificação de fetos maduros.

Em 2001, um artigo científico multicêntrico concluiu que os resultados fornecidos pelo teste de CCL são semelhantes aos métodos tradicionais de análise dos fosfolipídios, ressaltando que a vantagem mais atrativa do teste é o alto VPN. Este estudo também concluiu que, quando o resultado do teste é compatível com imaturidade, a chance de ocorrência de SDR é alta, estimada em 45,5\% dos $\operatorname{casos}^{20}$.

Os dados obtidos neste estudo, em concordância com outros autores ${ }^{12,13}$, demonstram que o teste da CCL apresenta sensibilidade, especificidade, VPP e VPN comparáveis aos testes de referência ${ }^{5,16,21-24}$, associado com a facilidade e rapidez na execução e baixo custo, podendo ser realizado rotineiramente na grande maioria dos centros e hospitais que disponham de um laboratório de análises clínicas com hematologia automatizada.

\section{Referências}

1. Dalence CR, Bowie L, Dohnal JC, Farrell EE, Neerhof MG. Amniotic fluid lamellar body count: a rapid and reliable fetal lung maturity test. Obstet Gynecol. 1995;86(2):235-9.

2. Hoyert DL, Kung HC, Smith BL. Deaths: preliminary data for 2003. Natl Vital Stat Rep. 2005;53(15): 1-48.

3. Dubin SB. Assessment of fetal lung maturity. Practice parameter. Am J Clin Pathol. 1998;1 10(6):723-32.

4. Grenache DG, Gronowski AM. Fetal lung maturity. Clin Biochem. 2006;39(1):1-10.

5. Karcher R, Sykes E, Batton D, Uddin Z, Ross G, Hockman E, et al. Gestational age-specific predicted risk of neonatal respiratory distress syndrome using lamellar body count and surfactant-to-albumin ratio in amniotic fluid. Am J Obstet Gynecol. 2005; 193(5): 1680-4.

6. Wijnberger LD, de Kleine M, Voorbij HA, Arabin B, Bruinse $\mathrm{HW}$, Visser $\mathrm{GH}$, et al. Prediction of fetal lung immaturity using gestational age, patient characteristics and fetal lung maturity tests: a probabilistic approach. Arch Gynecol Obstet. In press 2009.

7. Wijnberger LD, Huisjes AN, Voorbij HA, Franx A, Bruinse HW, Mol BW. The accuracy of lamellar body count and lecithin/sphingomyelin ratio in the prediction of neonatal respiratory distress syndrome: a meta-analysis. BJOG. 2001;108(6):583-8.

8. Souza E, Kobayashi S, Simões M, Camano L, Kulay Júnior L. Ação da betametasona em ratas prenhes: impacto sobre os níveis de corticosterona e glândulas adrenais maternas e fetais. Rev Bras Ginecol Obstet. 2001;23(10):667-73.

9. Cunningham FG, Gant NF, Leveno KJ, Gilstrap III LC, Hauth JC, Wenstrom KD. Hypertensive disorders in pregnancy. In: William JW, editor. Williams obstetrics. $21^{\text {st }}$ ed. New York: McGraw-Hill; 2001. p. 567-618.

10. Amorim MMR, Faúndes A, Santos LC, Azevedo E. Acurácia do teste de Clements para avaliação da maturidade pulmonar fetal em gestantes com doença hipertensiva específica da gestação. Rev Bras Ginecol Obstet. 1998;20(5):253-60.

11. Fernandes GL, Torloni MR, Klimke D, Hisaba WJ, Stivaletti J, Sancovski $M$, et al. Contagem de corpos lamelares versus teste de Clements na avaliação da maturidade pulmonar fetal em gestantes diabéticas. Rev Bras Ginecol Obstet. 2006;28(8):460-6.

12. Dubin SB. Characterization of amniotic fluid lamellar bodies by resistive-pulse counting: relationship to measures of fetal lung maturity. Clin Chem. 1989;35(4):612-6.
13. Abd El Aal DE, Elkhirshy AA, Atwa S, El-Kabsh MY. Lamellar body count as a predictor of neonatal lung maturity in high-risk pregnancies. Int J Gynaecol Obstet. 2005;89(1):19-25.

14. Ljubic V, Radunovic N, Stefanovic A, Opalic J. Quantitative analysis of lamellar bodies in amniotic fluid as fetal pulmonary maturity indicator. Vojnosanit Pregl. 2009;66(2): 113-5.

15. DeRoche ME, Ingardia CJ, Guerette PJ, Wu AH, LaSala CA, Mandavilli SR. The use of lamellar body counts to predict fetal lung maturity in pregnancies complicated by diabetes mellitus. Am J Obstet Gynecol. 2002; 187(4):908-12.

16. Haymond S, Luzzi VI, Parvin CA, Gronowski AM. A direct comparison between lamellar body counts and fluorescent polarization methods for predicting respiratory distress syndrome. Am J Clin Pathol. 2006; 126(6):894-9.

17. Richardson DK, Heffner L. Fetal-lung maturity: tests mature, interpretation not. Lancet. 2001;358(9283):684-6.

18. Lee IS, Cho YK, Kim A, Min WK, Kim KS, Mok JE. Lamellar body count in amniotic fluid as a rapid screening test for fetal lung maturity. J Perinatol. 1996;16(3 Pt 1):176-80.

19. Ashwood ER, Oldroyd RG, Palmer SE. Measuring the number of lamellar body particles in amniotic fluid. Obstet Gynecol. 1990;75(2):289-92.

20. Neerhof MG, Dohnal JC, Ashwood ER, Lee IS, Anceschi MM. Lamellar body counts: a consensus on protocol. Obstet Gynecol. 2001;97(2):318-20.

21. Bahasadri S, Changizi N. Association between lamellar body count and respiratory distress in neonates. Saudi Med J. 2005;26(9): 1414-6.

22. Ghidini A, Poggi SH, Spong CY, Goodwin KM, Vink J, Pezzullo JC. Role of lamellar body count for the prediction of neonatal respiratory distress syndrome in non-diabetic pregnant women. Arch Gynecol Obstet. 2005;271(4):325-8.

23. Neerhof MG, Haney El, Silver RK, Ashwood ER, Lee IS, Piazze JJ. Lamellar body counts compared with traditional phospholipid analysis as an assay for evaluating fetal lung maturity. Obstet Gynecol. 2001;97(2):305-9.

24. Ventolini G, Neiger R, Hood DL, Belcastro MR. Changes in the threshold of fetal lung maturity testing and neonatal outcome of infants delivered electively before 39 weeks gestation: implications and cost-effectiveness. J Perinatol. 2006;26(5):264-7. 